\title{
PA-145 ETHICAL AND SCIENTIFIC CONSIDERATIONS FOR THE DESIGN AND IMPLEMENTATION OF THE PREP DEMONSTRATION PROJECT IN NIGERIA
}

\author{
Morenike Ukpong, ${ }^{1,2}$ Atiene Sagay, ${ }^{3}$ Hadiza Khamofu, ${ }^{3}$ Kwasi Torpey, ${ }^{3}$ \\ Evaristus Afiadigwe, ${ }^{3}$ James Anenih, ${ }^{3}$ Oliver Ezechi, ${ }^{3}$ Chidi Nweneka, ${ }^{3}$ \\ John Idoko ${ }^{3}$. ' Obafemi Awolowo University, Nigeria; ${ }^{2}$ NHVMAS, Nigeria; ${ }^{3}$ Nigeria \\ PrEP Demonstration Study, Nigeria
}

\subsection{6/bmjgh-2016-000260.171}

Background This abstract highlights the ethical and scientific considerations that informed the development and review of the Nigeria PrEP demonstration study protocol.

Methods A desk review was conducted on all the meeting reports that led to the choice of the study design and the decisions made to modify the protocol for the PrEP demonstration project in Nigeria. The study focused on the ethical and scientific rationales for modifying the Partners' PrEP Sero-discordant protocol for this study as well as for the first and second protocol amendments of this study.

Results The decision to conduct a PrEP study was based on the outcome of a modelling study that suggested that serodiscordant couples will benefit from access to condom, TasP, and PrEP. Next, the decisions on the target population for the PrEP demonstration study, the models for evaluation at specific project site, and the design of the community engagement programme were reached through a formative research which engaged 611 individuals using multiple media. The study did not exclude study participants based on Hepatitis status and HIV risk profile since Truvada was an effective hepatitis treatment and the prevalence of hepatitis infection is high in Nigeria. Participants' interest in PrEP use was considered enough reason to prescribe PrEP in a country where uptake of ARV is slow and stigma associated with ARV use is high. Also, HIV-negative partners could assess when the viral load of the HIV-positive partner was 400 copies/ml. Since adherence was a challenge for PrEP use, adherence was enhanced through the use of the MEMS Cap.

Conclusions A PrEP demonstration study that mimics real life scenarios for PrEP provision within public health care institutions and is designed on the basis of community consultations, ethical and scientific considerations, will enhance the success of PrEP roll-out in resource-limited settings like Nigeria. 